\title{
“Update to Pseudobulbar affect (suddenly emotions of crying/laughing without control)"
}

\author{
Jorge Garza-Ulloa* \\ Research Consulting Services www.garzaulloa.org / University of Texas El Paso.
}

*Corresponding author: Jorge Garza-Ulloa, Research Consulting Services www.garzaulloa.org / University of Texas El Paso, US. To Cite This Article: Jorge Garza-Ulloa. "Update to Pseudobulbar affect (suddenly emotions of crying/laughing without control)". Am J Biomed Sci \& Res. 2021 - 13(1). AJBSR.MS.ID.001837. DOI: 10.34297/AJBSR.2021.13.001837.

Received: 眥 May 31, 2021; Published: 恝 June 09, 2021

\begin{abstract}
An "suddenly emotional" is when the simplest things like a simple comment or opinion of someone, a song, a memory, a TV commercial, a harsh word, or even a wayward perception, is all it takes for a person to be drowning in tears, or switch to an exaggerated laughing. These could be an indication of somebody could have "Pseudobulbar affect", this is a is a disorder that is characterized by episodes of sudden uncontrollable inappropriate laughing and/or crying. "Pseudobulbar affect" typically occurs in people with certain neurological conditions as brain disorders or injuries, which might affect the way the brain controls emotion. The "Pseudobulbar affect (PBA)" are emotions that leaves as fast as they come, making the affected person embarrassed when they spoke publicly, and they start crying or laughing. "PBA" is not really their fault, they have episodes that they cannot control, and they are not alone in this world, where millions of people with affected neurologic conditions have symptoms that may suggest the emotional disorders known as "Pseudobulbar affect". In this paper the goal is introduce you this disorder, explain the latest updates of how suddenly emotions of crying/laughing without control may suggest "Pseudobulbar affect", and point out the importance of document this kind of episodes of people that preset this behavior by ones around them, as : caregiver, their loved one, healthcare provider at palliative or hospice health care, all of this with the purpose to facilitate their analysis of the complexity of the symptoms that frequently are combined with neurological condition or brain injuries different by specialized doctors as neuropsychologist, neurologist, and psychiatrist to obtain a valid diagnostic in the less possible time to improve the quality of life of affected persons.
\end{abstract}

Keywords: Pseudobulbar affect, brain injury, Parkinson's disease, Parkinsonism, Parkinson's, Amyotrophic Lateral Sclerosis, Alzheimer dementia, Traumatic Brain Injury, Cerebrovascular disease, Multiple Sclerosis.

Abbreviations: (PBA): Pseudobulbar affect; (PD): Parkinson's disease; (CNS): central nervous system; (ALS): Amyotrophic Lateral Sclerosis; (ALZ): Alzheimer dementia; (TBI): Traumatic Brain Injury; (MS): Multiple Sclerosis; (CNS): central nervous system.

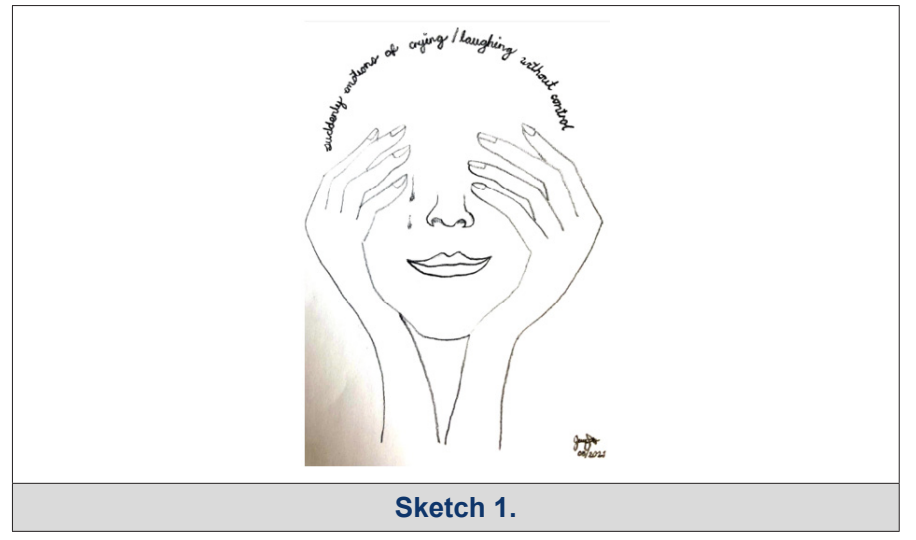

"suddenly emotions of crying/laughing without control" dedicated to my mom by Jeannie

\section{Introduction based on affected people com- ments and reports}

"Pseudobulbar affect" was first defined as a syndrome based on several symptoms occurring together and characterizing for specific disorders, that identifies irregularity or interruption of normal emotional functions expressed as crying and/or laughing without control. "Pseudobulbar affect abbreviated as PBA" typically occurs in people with certain neurological conditions or brain injuries, which might affect the way the brain controls emotions. This condition might interfere with abilities to work and do daily tasks, especially when already the affected one is handling other symptoms from neurologic conditions or brain injuries. Usually "PBA" causes embarrassment, social isolation, stress, anxiety, sleep 
disorders and even depression for affected patients.

Many millions of affected people around the world report symptoms that may suggest "Pseudobulbar affect" without being aware of their current neurologic condition or brain injuries. They frequently report with comments like the following:

- $\quad$ "My emotions leave me as fast as they come. They are not real or mine like they used to be."

- $\quad$ "I cry now a lot; I have never been a crier...all the time."

- $\quad$ "Sometimes when I have spoken publicly, I will start crying or laughing. Very embarrassing!! I therefore don't do any public speaking anymore".

- $\quad$ "I cry frequently at TV commercials, and I cannot stop for minutes."

- "I wait till everyone is out of the movie, so i can go out without people seeing my tears. I feel silly."

- $\quad$ "My eyes used to become tearful on any sad news or situation. I did not think at all that this may be due to underline depression."

And more many similar reports of episodes of sudden uncontrollable and inappropriate laughing or crying at inconvenient moments.

"Caregivers and their loved one of people with neurological condition or brain injuries" also report their experiences as a wide feeling when receive suddenly changing emotions from the patients all days long as:

- "Guilty about no knowing how to help them",

- "Confused and helpless wondering where the crying or laughing are coming from",

- "Sadness at seeing their patients struggling with sudden emotion that impact their quality of life".

- And many similar reports

"Healthcare provider at palliative or hospice health care providers" frequently are challenged to make a diagnosis and treatment plan with lack of education on "pseudobulbar affect (PBA)", leading them to ineffective treatments based on wrong clinicals impressions identifying the causes as: "stress", "pain", "anxiety", sleep disorders", "depression", "bipolar disorder" and many others. Where:

- $\quad$ "Stress" defined as a physical, mental, or emotional factor that causes bodily or mental tension. It can be external from the environment, psychological, or social situations or internal based on illness, a medical procedure, pain, etc. $[1,2]$.

- " "Pain" is defined as physical suffering or discomfort caused by illness or injury [3]
- "Anxiety" as an emotion characterized by physical manifestation of stress, worried thoughts that lead to excessive nervousness, fear, apprehension, and inclusive physical symptoms changes as increased blood pressure and others that may seriously affect day-to-day living [1], "anxiety" is a common manifestation in depressive states of the affective disorders [4]

- "Sleep disorders" are frequently initiated with "sleep changes patterns" due to a big diversity of reasons as: "stress", "anxiety", aging, hormonal levels changes, mood, sleep apnea, snoring, lifestyles, environment changes, restless leg syndrome [43], and many other reasons. "Sleep" is defined as the normal condition of body and mind such as that which typically recurs for several hours every night, in which the nervous system is relatively inactive, the eyes closed, the postural muscles relaxed with consciousness practically suspended and the normal "Sleep stages", which is duration is different at various age [1]. All of this based on changes in sleep patterns in older men have been linked to an increased risk of cognitive decline [5]. Until recently, both the purpose of the biological need for sleep and the mechanism by which the "central nervous system (CNS)" eliminated metabolic waste products were unknown. The "glymphatic system" is the recently discovered macroscopic waste clearance system for the "CNS", because it rushes along right next to the brain's blood vessels, delivering key supplies while clearing away unwanted debris. It which predominantly functions during sleep states [6]

- "Depression" as is a constant feeling of sadness and loss of interest, which stops you doing your normal activities. Different types of "depression" exist, with symptoms ranging from relatively minor to severe. Generally, depression does not result from a single event, but from a mix of events and factors [7]. For example: "Surviving cardiac arrest" is characterized by cognitive, psychological, and functional impairments and apprehension over long-term prognosis. A first estimated the proportion of clinically significant depression and anxiety in cardiac arrest survivors and their informal caregivers is bigger than previous thought [8].

- "Bipolar disorder" formerly called "manic-depressive illness or manic depression" is defined as a mental disorder that causes unusual shifts in mood, energy, activity levels, concentration, and the ability to carry out day-to-day tasks [9]. "Bipolar disorder" is associated with increased rates of separate physical diseases likely involving inflammatory components in the pathogenesis including ischemic heart disease, diabetes, dementia, hypertension, hypercholesterolemia and hyperlipidemia, hypothyroidism, and infections [10].

- And others clinicals impressions based of the intense emotions experienced by "Pseudobulbar affect", taking them to take wrong decisions to health care provider at palliative or hospice 
health care providers, caregivers and their loved one can easily misjudged when they try to help their patients. Although an estimated $9 \%$ of nursing home residents have symptoms suggestive of PBA, they are not routinely screened [11].

\section{Pseudobulbar Affect (PBA) Best Definition}

Charles Darwin since 1872 described about "involuntary laughing and crying as pseudobulbar palsy patients" [12], and Oppenheim in 1911 [13] was the first to use the term "pseudobulbar affect", and Wilson in 1924 [14] defined "Pseudobulbar affect (PSA) as a stereotype emotional display with characteristically uncontrollable, involuntary outburst of laughing or crying that mimic an emotional expression that does not reflect the actual appropriate mood status of the patient".

Actually, "Pseudobulbar affect" is identified as:

a) "PBA or pathologic laughing/crying is defined as a disorder based on the disturb of normal function for emotional expressions, and not only as a syndrome based on collection of signs and symptoms".

b) "PBA is distinguishable from mood disorders in which emotional expressions are associated with actual mood or feelings" [15].

c) "PBA" is a disorder of emotional expression rather than a primary disturbance of feeling, that can cause a significant burden of illness in patients already impacted by a serious neurologic disease, including impairments in overall health status" [16]

Because "PBA" symptoms always happen when certain "neurologic conditions or brain injuries" exist, it is recommended to be treated only by a specialist doctor who can diagnose "pseudobulbar affect identified (PBA)" as neuropsychologist, neurologist, and psychiatrist. It is often detected by theses expert clinicians in an informal manner, as a part of their routine neurological evaluations. The most frequently neurological diseases that could include "PBA" are: "Amyotrophic Lateral Sclerosis", "Alzheimer dementia”, "Traumatic Brain Injury (TBI)", "Cerebrovascular disease", "Multiple Sclerosis", "Parkinsonism", "Parkinson's Disease (PD)", and others. Where:

a) "Amyotrophic Lateral Sclerosis (ALS)" is a progressive nervous system disease that affects nerve cells in the brain and spinal cord, causing loss of muscle control. "ALS" is often called "Lou Gehrig's disease", after the baseball player who was diagnosed with it. Doctors usually do not know why "ALS occurs" but some cases are inherited [17].

b) "Alzheimer dementia" is a progressive neurodegenerative disease that occurs when nerve cells in the brain die, where dementia symptoms gradually worsen over several years. In its early stages, memory loss is mild, but with late stage "Alzheimer's", individuals lose the ability to carry on a conversation and respond to their environment. "Alzheimer's" is the most common cause of dementia [18], where "dementia" is defined as a loss of mental skills, such as memory, cognition, problem solving, and learning severe enough to interference with daily life. There are many kinds of dementia and "PBA" could be present in many of them [19]

c) “Traumatic Brain Injury (TBI)" usually results from a violent blow or jolt to the head or body. An object that goes through brain tissue, such as a bullet or shattered piece of skull, often occurs because of a severe sports injury or car accident. "Mild traumatic brain injury" may affect your brain cells temporarily. More "serious traumatic brain injury" can result in bruising, torn tissues, bleeding, and other physical damage to the brain. These injuries can result in long-term complications or death $[20,21]$.

d) "Cerebrovascular disease" refers to a group of conditions, diseases, and disorders that affect the blood vessels and blood supply to the brain. If a blockage, malformation, or hemorrhage prevents the brain cells from getting enough oxygen, brain damage can result. "Cerebrovascular diseases" include "stroke", "transient ischemic attack (TIA)", "aneurysm", and "vascular malformation" [22].

e) "Multiple Sclerosis (MS)" is a potentially disabling neurological progressive disease of the "brain and spinal cord (central nervous system)". Where, the immune system attacks the protective sheath (myelin) that covers nerve fibers and causes communication problems between your brain and the rest of your body $[23,24]$.

f) "Parkinsonism" is a constellation of signs and symptoms that are characteristically observed in "Parkinson's disease (PD)", but that are not necessarily due to PD $[25,26]$.

g) "Parkinson's Disease (PD)" is a progressive neurodegenerative disease producing neuronal cell death, presenting loss of dopamine production in the brain area known as "substancia nigra", altering the "central nervous system CNS" formed by brain and spinal cord, and affecting the regulation of the human movements and emotions $[27,28]$

And others neurological diseases that could or could not include "PBA" disorder.

\section{Pseudobulbar Affect Must be Evaluated by Neu- rological Disorders Specialists}

The neurologist Dr. Klaus Poeck in 1969 [29] defined the basic 4 criteria for detection of "PSA" as: 
1) When the emotional response is situationally inappropriate,

2) Patient's feelings and the affective response are not closely related,

3) The duration and severity of the episodes cannot be controlled by the patient, and

4) Expression of the emotion does not lead to a feeling of relief.

Then, Dr. Jeffrey L. Cummings et al. in 2006 [30] defined 6 criteria for detection of "PBA" as:

1) Changes from previous emotional responses,

2) Inconsistent with or disproportionate to mood,

3) Not dependent on a stimulus or are excessive relative to that stimulus,

4) Cause significant distress or social/occupational impairment,

5) Not accounted for by another psychiatric or neurologic disorder and

6) Not due to a drug.

The most frequently PBA diagnostic criteria are from [30,31]: "The Center for Neurologic Study-Liability Scale (CNS-LS) and the Pathologic Laughter and Crying Scale (PLACS)". Where:

- $\quad$ The Center for Neurologic Study-Liability Scale (CNS-LS) [32] is a short 7-item, self-administered questionnaire designed to be completed by the patient, that provides a quantitative measure of the perceived frequency of PBA episodes. Add the values for each of the 7 items to get the total score evaluating the answer with the following 5 options: Never 1 point, Rarely $=2$ points, Occasionally $=3$ points, Frequently $=4$ and Most of the time $=5$ points. "A CNS-LS score of 13 or higher may suggest PBA*". The CNS-LS can help accurately diagnose PBA. The CNS-LS 7 items used for autoevaluations; these are:

I. If there are times when I feel fine one minute, and then I will become tearful the next over something small or for no reason at all.

II. Others have told me that I seem to become amused very easily or that I seem to become amused about things that really are not funny.

III. I find myself crying very easily.

IV. I find that even when I try to control my laughter, I am often unable to do so.

V. There are times when I will not be thinking of anything happy or funny at all, but then I will suddenly be overcome by funny or happy thoughts.

VI. I find that even when I try to control my crying, I am often unable to do so.

VII. I find that I am easily overcome by laughter.

- $\quad$ "The Center for Neurologic Study-Liability Scale (CNS-LS) and the Pathologic Laughter and Crying Scale (PLACS) [33] have a more precise test than consists of 18 questions and must be carried out by a health professional*. Scores for each question range from zero (normal) to three (excessive emotional lability). It looks at several aspects of "PBA" including duration of, and the extent of distress following, an episode. This scale has not been validated in people with "Multiple Sclerosis (MS)", but it has been used in "MS studies of PBA". A score of 13 or more would indicate presence of "PBA".

Note*: It is important to get a prompt and accurate diagnosis of "pseudobulbar affect" by specialist doctors as neuropsychologist, neurologist, and psychiatrist to enable you to access appropriate treatment, as well as support for you and your family. Counselling may be helpful to reassure the patient and their families that "PBA is not the fault of the patient". 


\section{Pseudobulbar Affect Differences with Depres- sion}

Because "PBA" patient's emotional response is noted as disproportionate and, therefore, overlooked as "depression", or changes are attributed to the patient's baseline neurocognitive disease. Often, patients are misdiagnosed as having "depressed mood". Based on different research report [31-34,13]:

- In average from $30 \%$ to $35 \%$ of patients with "PBA" are "depressed".

- "The duration and type of symptoms can help delineate depression from PBA".

- $\quad$ "Depression tends to have a longer clinical course, whereas PBA is often of a brief nature with episodic occurrences".

- $\quad$ "The exaggerated response of patients with PBA, as well as the discordant response, is also exclusive to PBA".

- Neurovegetative symptoms such as sleep, and appetite disturbances are relegated to "depression" and are not associated with "PBA" It is very import to mention the "PBA" appears to be related with the "Theory of Stress-Anxietysleep disorders -Neural Damage Chain in neurological diseases and brain damage" [1] as explained in this research article with concepts involved, many reasons to be included in the theory and their relations to previous conclusions/ suggestions of many researchers' papers already publish and recommendations to develop good habits in neurological patients to minimize the "PBA" disorder as explain in a real case in section of this research: "section 8 Testimony to diagnose pseudobulbar affect"

\section{What Causes Pseudobulbar Affect?}

In general, "brain damage" is the cause of Pseudobulbar affect (PBA)", it could be from:

- "Stroke" when a blood vessel in the brain is blocked or bursts. Without blood and the oxygen, it carries, part of the brain starts to die. Then the part of the body controlled by that area of the brain cannot work properly.

- $\quad$ "Brain tumor" is a collection, or mass, of abnormal cells in your brain. The skull, which encloses your brain, is very rigid. Any growth inside such a restricted space can cause problems. Brain tumors can be cancerous (malignant) or noncancerous (benign).

- "Neurological disorders" related to neurological disorders" mainly: "Amyotrophic Lateral Sclerosis", "Alzheimer dementia”,
"Traumatic Brain Injury (TBI)", "Cerebrovascular disease”, "Multiple Sclerosis", "Parkinsonism”, "Parkinson's Disease (PD)", and others. As explained at "section 3 Pseudobulbar affect must be evaluated by neurological disorders specialists.

\section{Pathophysiology on Pseudobulbar Affect}

The "Pathophysiology" referrers to the study of abnormal changes in the human body functions that are the causes, consequences, or concomitants of a disease process. The "Pathophysiology on Pseudobulbar affect (PBA)" identify a lack of voluntary control in the "neurons" as specialized cell known as the basic unit of the "human nervous system", these "neurons" cells as shown in figure 1 a make a up a massive network of "specialized nerve cells" connected with the purpose of transmit messages very rapidly, from one part of the body to another, creating complex interconnected circuits known as: "neural pathways". They are "clusters of neurons" connections from one part of the "nervous system" to another using union between them identified as "synapses" as shown in figure $1 \mathrm{~b}$. The brain under the right conditions can change hundreds of millions and possibly billions of the connections between the nerve cells [35] in "neural pathways" as shown in fig $1 \mathrm{c}$, every time we learn, react, process and store new information in the brain or send/receive orders to the nervous system.

All the neurons transmit signals of mainly two kinds "electrical" and "chemical". Where:

"Electrical signals are inside the neurons" and "chemical signals are between neurons through the synapse space" indicate by blue circles at figure $1 \mathrm{~b}$ and c. The "synapse chemical activity" trigger the release of "neurotransmitters" which carry the impulse across the "synapse" to the next "neuron". Once a nerve impulse has triggered the release of "neurotransmitter", these chemical messengers also known as "hormones" cross the tiny "synaptic space" and taken up by specialized receptors on the surface of the next cell. "This process converts the chemical signal back into electrical signal", if the signal is strong enough, it will be propagated down to the next neuron until once again reaches another "synapse" and the process is repeated once more, and so on until reach the destination as shown in (Figure 1) c.

The main point to explain the "PBA" disorder is that different "neurotransmitters" fulfill different functions in the brain as: "excitatory" and "inhibitory". Where:

- "Excitatory neurotransmitters" acts stimulating the firing in the "synapse", and

- "Inhibitory neurotransmitters" tend to block it in the responding cell. 


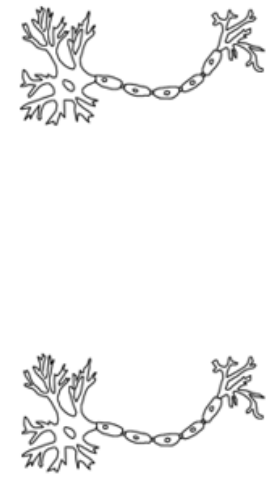

a) Two separated "neurons"

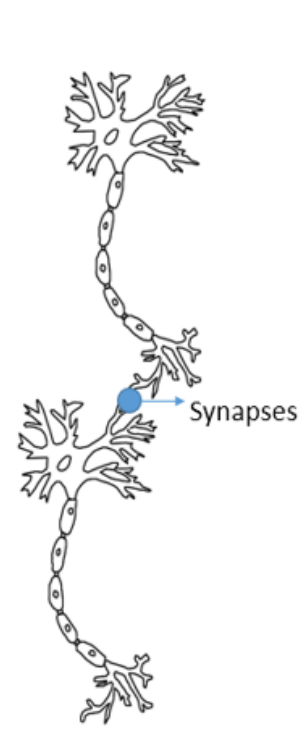

b) Two connected "neurons" trough union named "synapses"

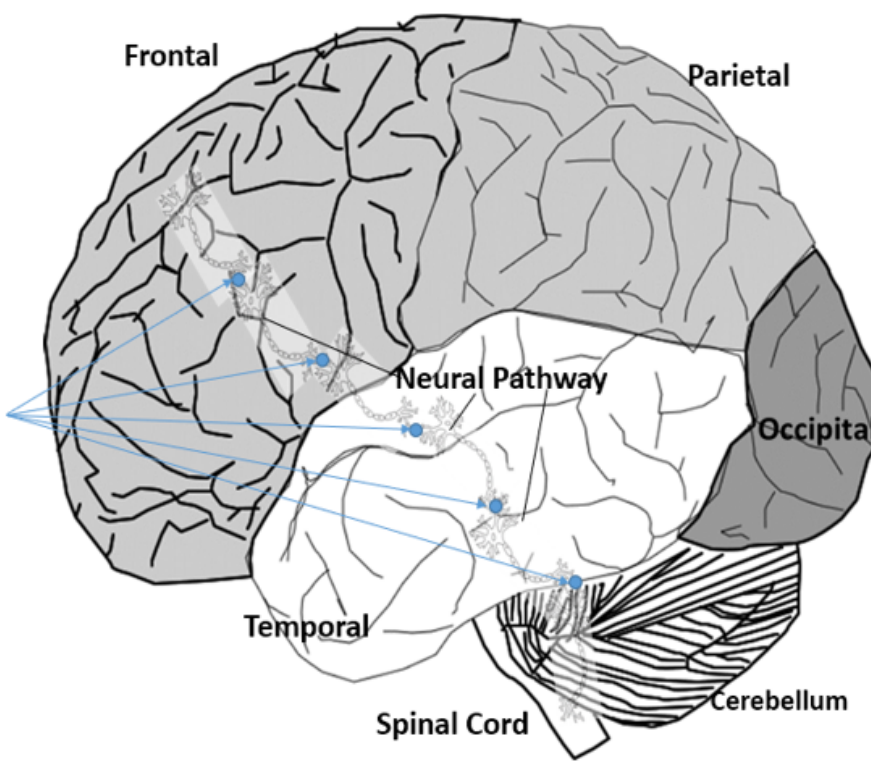

c) A brain "neural pathway" connecting "frontal", "temporal" "Cerebellum" to "Spinal Cord". The "synapses" are indicated in each union between neurons

Figure 1: a) Two separated "Neurons", b) Two Connected "Neurons" Trough "Synapse", and c) A brain "Pathway"'

When the signal reaches its destination is the results of the combination between "Excitatoryand Inhibitory neurotransmitters" in each "synapse" in the complete "pathway". There are different types of small molecules manufactured in different kind of axons that are in the tail of each neuron, known generally as "neurotransmitters" [1], some of them are: "acetylcholine", "dopamine", “GABA", "glutamate", "glycine", "norepinephrine", "epinephrine or adrenaline", "serotonin", and many more. Where:

- "Acetylcholine" has an "excitatory neurotransmitter function" is used by the "spinal cord" to cause "muscle contraction", and many neurons in the brain to regulate memory.

- "Dopamine" has "both inhibitory and excitatory neurotransmitter functions" depending upon where in the "brain and which receptor site it binds to". "Dopamine" plays a mayor role in regulate movement, attention, learning, and emotional responses.

- "GABA" has an "inhibitory neurotransmitter function". It is important to produce sleep, reducing anxiety, and forming memory.

- "Glutamate" has an a powerful "excitatory neurotransmitter function". It is important in learning and memory.

- "Glycine" has a "inhibitory neurotransmitter function". It is used mainly by the neurons in the "spinal cord".

- "Norepinephrine" has "inhibitory and excitatory neurotransmitter functions". It is used to regulate blood pressure and calmness.
- "Epinephrine or adrenaline" has "excitatory neurotransmitter function" in the "peripheral nervous system" and "inhibitory neurotransmitter function" in a few brain areas. The Key actions of "adrenaline" include increasing the heart rate, increasing blood pressure, expanding the air passages of the lungs, enlarging the pupil in the eye, redistributing blood to the muscles, and altering the body's metabolism, with the purpose of maximize blood glucose levels primarily for the brain.

- "Serotonin" has "inhibitory neurotransmitter function". It is a complex and multifaceted, modulating mood, cognition, reward, learning, memory, and numerous physiological processes. Low levels of "serotonin" in the brain may cause depression, anxiety, and sleep trouble.

And many other neurotransmitters types are used in the synapses in the neural pathways.

There are an estimated of 100 billon "neurons" making an approximation of 100 trillion "neural connections". These process information in the brain, and they can send orders to "body movements" known as "motor pathways" and receive information from "humans' senses" known as "sensory pathways", "the pathways are so complex and are not completely understood". Besides store memories, update them, new ones from new ideas based on creativity or reconnection of old information stored. Detailed reviews of the widespread anatomical and neurophysiological abnormalities found by Neuroimaging and neurophysiological studies in patients with "Pseudobulbar affect (PBA) disorder seems 
to indicate that the neuronal paths shown unbalanced between the inhibitory and excitatory neurotransmitter functions in the synapses involved in the pathways" [46].

The "primary neurotransmitters" believed to be involved in "PBA" are "serotonin" and "glutamate". Based on the role of "serotonin as inhibitory neurotransmitter function in corticolimbic or cerebellar pathways" may account for its impact on this disorder. "Glutamate" as an "excitatory neurotransmitter function" that is present in over $90 \%$ of all "brain synapses", whose receptors are widely distributed within the brain. Thus, bases on clinical research:

- The areas of the brain that control the expression of emotion, such as the "prefrontal cortex" are frequently affected on patients with "PBA" [36].

- The alterations in chemicals in the brain that contribute to the development of the condition. Disrupting brain signaling, triggering episodes the person affected cannot control, the most common are "In the synapses the modulation of glutamatergic and serotonin transmission" [37]. These unbalance on different "synapses on brain pathways" can have widespread effects, and that are affected by "brain damage" as explained in this paper "section 5 What causes PBA? " [47 ]

- Other researches had concluded: "In the brain pathways the cerebellum appears to play a far larger role in PBA". There are pathways from the "cortex to pons to cerebellum that appear to control not only motor, but also cognitive and affective function" [29]

- It likely results from the compromise of a network of descending pathways, specifically the "cortico-pontinecerebellar circuit" as one of the largest pathways associated with the "cerebellum". Arising in the cerebral cortex these fibers first terminate ipsilaterally in the pontine nuclei. Then the fibers decussate and form the middle cerebellar peduncle, terminating in the cerebellar cortex as mossy fibers, and/ or dysfunction in certain neurotransmitters, particularly "serotonin and glutamate" [16].

\section{Pseudobulbar Affect Strategies and Treat- ments}

There is not known cure for "Pseudobulbar affect (PBA)", but it has been proving the severe complications related as embarrassment, social isolation, anxiety, and depression. The condition might interfere with your ability to work and do daily tasks, especially when you are already coping with a neurological condition. Besides, medications can reduce the number of "PBA" episodes or make them less intensive.

"Lifestyle strategies may help manage PBA" as:
- $\quad$ Keeping a diary to track what triggers the episodes and sharing it with a doctor.

- Also, when a person feels an episode approaching, he or his/ her caregiver may try to focus on something unrelated and adjust his posture.

- Taking slow, deep breaths during an episode until they are in control also may help, and so can relaxing the forehead, shoulders, and body.

- $\quad$ Build a healthy environment free of worries to the affected people like avoiding everyday impact news, staying away from pessimistic people that usually speak about bad news, stop given to much importance to "PBA episodes", etc.

"The goal of treatment of PBA is no to cure but focus into diminish the severity and frequency of the episodes". This is achieved targeting the primary "neurotransmitters" that affect cognition and emotions as "norepinephrine", "serotonin", or "glutamate" allows under specialized medical recommendation and supervision as "antidepressants", "Selective serotonin reductase inhibitors (SSRIs)", "Cough suppressant dextromethorphan", "Dopaminergic medications" and others. Where:

- "Antidepressants" as "Tricyclic antidepressants (TCAs)" [37] for the treatment of "PBA" are typically prescribed at doses lower than are those used to treat depression [38].

- "Selective serotonin reductase inhibitors (SSRIs)" [39] these are a class of drugs that are typically used as antidepressants in the treatment of major depressive disorder, anxiety disorders, and other psychological conditions.

- “Cough suppressant dextromethorphan" [38]. This time, the only treatment currently approved by the U.S. Food and Drug Administration (FDA) for the treatment of "PBA" is an oral medicine that contains "dextromethorphan hydrobromide and quinidine sulfate". The active ingredients are dextromethorphan hydrobromide monohydrate USP and quinidine sulfate dihydrate USP [39][40][48]

- "Dopaminergic medications" such as "levodopa and amantadine" have been used as well, but with lower response rates [41]

\section{Testimony to Diagnose Pseudobulbar Affect}

I am a caregiver of a family member with "Parkinsonism: Atypical Parkinson" neurological disease as a both a chronic and progressive movement. After 5 years her Parkinsonism: Atypical Parkinson" was identified as: "Progressive Supranuclear Palsy (PSP)" is the most common degenerative type of atypical parkinsonism. Symptoms tend to progress more rapidly than "PD". People with "PSP" may fall frequently early in the course of disease. 
Later symptoms include limitations in eye movements, particularly looking up and down, which also contributes to falls. Those with "PSP" also often have problems with swallowing (dysphagia), difficulty in producing speech (dysarthria), sleep problems, memory and thinking problems (dementia). Its average age of onset is in the mid-60's.

Where was notorious the frequently crying since the beginning of her Parkinson's, usually without any apparent reason, unfortunately the specialized doctors took 10 years from the beginning of her disease to correctly identified the additional disorder as "pseudobulbar affect (PBA)", probing that is extremely hard to detect correctly besides her neurological progressive disease even for specialized doctors. Consulting different specialized doctors that were following the way of diagnoses her suddenly crying as different conclusions: "Stress-Anxiety", "Neuropathic Pain", "sleep disorders", and "Depression". Where the treatments and results were:

"Stress" as a response to a threat in a situation and "anxiety" is a physical reaction to the "stress" [1].

- They recommended treatment based on: A comfortable family environment was built, free of worries and problems of any kind to reduce her "anxiety" and by consequence her "stress", with a lot of personal care, love, and comprehension.

- Result: It helped to reduce it, but the "crying" episodes continued.

"Neuropathic Pain" is often described as a burning or shooting pulses, she suffered of numbness and tingling, and frequently feel pain from a touch that would not normally be painful. In her specific case was diagnosed to mainly "stiff muscles" complaint.

- $\quad$ Recommended treatment: "Frequently rest" was implemented because the persons with neurologic disease uses more energy to do anything that a normal people, and they frequently feel very tired. Periodic "muscles massage" to increase relaxation, reduce pain improve blood circulation and alertness, and "applications of heat or cold" base in the fact that "heat work" is better for muscle tightness and "cold" may work better for swelling and inflammation. The options were to apply hot and cold packs, heating pads, heat therapy patches and diclofenac sodium topic gel.

- Result: It helped to reduce it a little more, but the "crying" episodes continued.

"Sleep disorders" in neurological disorders is frequently suspect the accumulated actions from years to of "stress" and "anxiety" plus others factors as: aging, hormonal levels changes, mood, sleep apnea, snoring poor lifestyle environment changes, etc.
- Recommended treatment: In her case the sleep cycles in quality and duration presented a "Rapid Eye Movement behavior disorder" characterized by "dream enactment (acting while dreaming)" where the muscle paralysis that normally occurs during "REM Sleep stages" was incomplete or absent [40]. She also presented "Rest less syndrome" as a condition that causes an uncontrollable urge to move your legs during resting [43].

- Recommended treatment: "Ramelteon as hypnotics melatonin M1/M2 Receptor agonists", but it did not work, then a prescription of an antidepressant that belongs to a group of drugs called "serotonin receptor antagonists and reuptake inhibitors (SARIs)". She only achieved to sleep 2 o 4 hours every night, the final prescription was a "Sedative-Hypnotic Benzodiazepines", and finally it works achieving 7-8 hours of sleep.

- $\quad$ Result was that the suddenly crying diminish about $33 \%$ in total.

"Depression" as a continuous symptom as: feelings of sadness, feelings of hopelessness, loss of interest/lack of pleasure, short temper, irritation, tiredness, memory loss, sleep disorders, crying uncontrollably, reduced appetite and weight Loss, etc.

- $\quad$ Recommended treatment: Antidepressants to relax the mind and Psychotherapy •

- Result: was that the suddenly crying diminish a little more about $40 \%$ in total.

After 10 years of visiting different specialized neurologists, one detected her disproportionate Patient's emotional response in an episode as "Pseudobulbar affect (PSA)" as described in this research under a normal evaluation appointment.

- $\quad$ Recommended treatment contains "dextromethorphan hydrobromide and quinidine sulfate".

- $\quad$ Result: was that the suddenly crying diminish a little more about $70 \%-80 \%$ in total.

\section{Pseudobulbar Affect Conclusions}

"Pseudobulbar affect (PBA)" is a nervous system disorder that is reflect with suddenly emotions of crying/laughing without control and it is related with: brain injuries including stroke, and neurological disorders based in the following conclusions:

- "Pseudobulbar affect (PBA)" affects the areas of the brain that control the expression of emotion, such as "prefrontal cortex", there also maybe alterations in the "chemical neurotransmitters" that contribute the development of this condition. These alterations may disrupt brain signaling, triggering episodes that the person affected cannot control. 
- "Pseudobulbar affect (PBA)" is often unrecognized and misdiagnosed. Patients with "PBA" experience episodes of spontaneous or exaggerated laughter and/or crying that are disproportionate to or inappropriate for the current situation. It is often mistaken for mental health conditions such as depression and hidden by the symptoms of the commonly associated neurologic diseases mainly: "Amyotrophic Lateral Sclerosis", "Alzheimer dementia (AD)", "Traumatic Brain Injury (TBI)", "Cerebrovascular disease", "Multiple Sclerosis", "Parkinsonism", “Parkinson's Disease (PD)", and others

- "Caregivers has a substantial burden when caring for patients with PBA" compared with caregivers of patients with the same underlying neurological condition but without "PBA" that affects patients [44].

- "PBA can be managed with pharmacologic intervention" including Antidepressant medicine, usually in a lower dose than for depression or Dextromethorphan and quinidine as the first line drug of choice for PBA [45, 31].

- "PBA" in nursing home residents was associated with chart documentation of uncontrollable crying, presence of a neurologic disorder, or by the documented presence of at least 2 of the following: stroke, severe cognitive impairment, and schizophrenia [11]

- "At this time has been find that several brain pathways contribute to laughter and crying in PBA", each with different brain areas and component to achieve the emotion that control our behavior to be "inhibited or excited" to facilitate the correct balance by "neurotransmitter" on the connection between neurons identified as "synapses."

This research paper main objective is to diminish the time to obtain the correct diagnose of "Pseudobulbar affect (PBA)" making a general observations to document the typical symptoms from the patients by caregiver, their loved one, healthcare provider at palliative or hospice health care providers to facilitate different specialized doctors as neuropsychologist, neurologist, and psychiatrist to obtain a valid diagnostic beside the complexity of symptoms combined neurological condition or brain injuries in the less possible time to improve the quality of life of "PBA" patients.

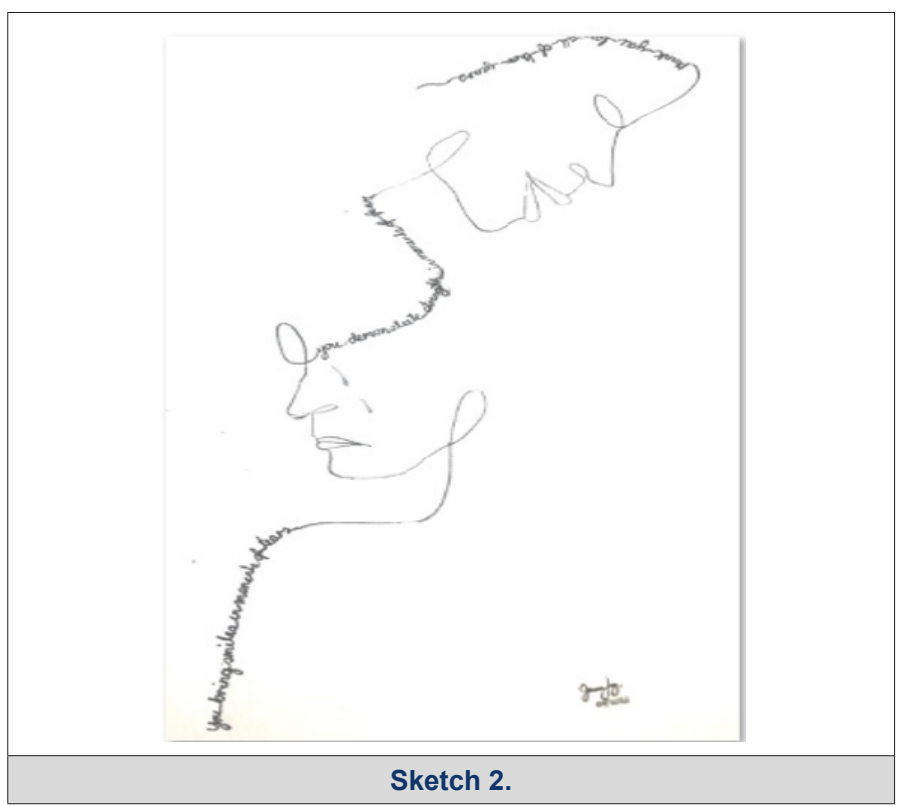

"You bring me smiles at the moments of tears, you demonstrate strength in moments"

\section{References}

1. Jorge Garza Ulloa (2019) Theory of Stress-Anxiety-Sleep DisordersNeural Damage Cyclic Chain and the Progression of Parkinson's Disease. Am J Biomed Sci \& Res 6(3).

2. Cordelia Gray, Alex Calderbank, Joy Adewusi , Rhiannon Hughes, Markus Reuber (2020) Symptoms of posttraumatic stress disorder in patients with functional neurological symptom disorder. J Psychosomatic Res 129: 109907

3. Victor C Wang, William J Mullally (2020) Pain Neurology. Am J Med 133(3): 273-280.

4. Malcolm Lader, Issac Marks (1971) Chapter Three - CLINICAL ANXIETY, Editor(s): MALCOLM LADER, ISSAC MARKS, Clinical Anxiety, Butterworth-Heinemann 22-49.

5. Jesse L Parker, Sarah L Appleton, Yohannes A Melaku, David Stevens, Gary A Wittert, et al. "Sleep macroarchitecture but not obstructive sleep apnea is independently associated with cognitive function in only older men of a population-based cohort". J Sleep Res 13370.

6. Jennaya Christensen, Glenn R Yamakawa, Sandy R Shultz, Richelle Mychasiuk (2021) Is the glymphatic system the missing link between sleep impairments and neurological disorders? Examining the implications and uncertainties. Prog Neurobio 198: 10191.

7. ht tps: / / w w w.betterhealth.vic.gov.au / he alth / conditionsandtreatments/depression], 05/04/2021. 
8. Alex Presciutti, Mary M Newman, Ana Maria Vranceanu, Jonathan A Shaffer, Sarah M Perman (2020) Associations between depression and anxiety symptoms with quality of life in cardiac arrest survivors with good neurologic recovery and informal caregivers of cardiac arrest survivors,Journal of Affective Disorders Reports, 100046.

9. https://www.mayoclinic.org/diseases-conditions/bipolar-disorder/ symptoms-causes/syc-20355955, 05/06/2021

10. Lars Vedel Kessing, Simon Christoffer Ziersen, Per Kragh Andersen, Ma Vinberg (2021) A nation-wide population-based longitudinal study mapping physical diseases in patients with bipolar disorder and their siblings. J Affect Disord 282: 18-25.

11. Carrie Allen, Barbara Zarowitz, Terrence O Shea, Edward Peterson, Charles Yonan, et al. (2018) Identification of pseudobulbar affect symptoms in the nursing home setting: Development and assessment of a screening tool. Geriatr Nurs 39(1): 54-59.

12. Hess U, Thibault P (2009) Darwin and emotion expression. Am Psychol $64(2): 120-128$.

13. Parvizi J (2009) Pathological laughter and crying. In: Savitz SI, Rontha M, eds. Neurology review for psychiatrists. Philadelphia: Lippincott Williams and Wilkins, 68-72.

14. Wilson SAK (1924) Some problems in neurology. II: pathological laughing and crying. J Neurol Psychopathol 4: 299-333.

15. Parvizi J, Coburn KL, Shillcutt SD (2009) Neuroanatomy of pathological laughing and crying: a report of the American Neuropsychiatric Association Committee on Research. J Neuropsychiatry Clin Neurosci 21(1):75-87.

16. Reus and D. Lindqvist (2019) Handbook of Clinical Neurology,Vol. 165 (3rd series)Psychopharmacology of Neurologic Disease V.I. Editorshttps://doi.org/10.1016/B978-0-444-64012-3.000149Copyright (C) 2019 Elsevier B.V

17. https://www.mayoclinic.org/diseases-conditions/amyotrophic-lateralsclerosis/symptoms-causes/syc-20354022

18. https://www.alz.org/alzheimers-dementia/what-is-alzheimers, $05 / 04 / 2021$

19. https://www.alz.org/alzheimers-dementia/what-is-dementia/typesof-dementia, 05/06/2021

20. https://www.mayoclinic.org/diseases-conditions/traumatic-braininjury/symptoms-causes/syc-20378557, 05/04/2021

21. A Obenaus, Loma Linda (2016) Encyclopedia of Mental Health (Second Edition), Traumatic Brain Injury Elsevier Inc.

22. https://www.medicalnewstoday.com/articles/184601

23. https://www.mayoclinic.org/diseases-conditions/multiple-sclerosis/ symptoms-causes/syc-20350269, 05/042021.

24. Maria K. Houtchens, Samia J. Khoury (2013) in Women and Health (Second Edition).

25. Jorge Garza-Ulloa (2019) A Parkinson's Disease Journey from Patient side view. Am J Biomed Sci \& Res 5(1).

26. Alice Hosking, Adrianus A L J Hommel, Stefan Lorenzl, Miguel Coelho, Joaquim J Ferreira, et al. (2021) Characteristics of Patients with LateStage Parkinsonism Who are Nursing Home Residents Compared with those Living at Home. Journal of the American Medical Directors Association 22(2): 440-445.

27. Jorge Garza-Ulloa (2019) Update on Parkinson's Disease. Am J Biomed Sci \& Res 2(6): AJBSR.MS.ID.000614

28. Nutan Sharma (2008) in Essentials of Physical Medicine and Rehabilitation (Second Edition), 2008
29. Poeck K (1969) Pathophysiology of emotional disorders associated with brain damage. In: Vinken P, Bruyn G, eds. Handbook of clinical neurology 3: $343-367$

30. Jeffrey L Cummings, David B Arciniegas, Benjamin R Brooks, Robert M Herndon, Edward C Lauterbach, rt al. (2006) Defining and diagnosing involuntary emotional expression disorder. CNS Spectr 11(6):1-7.

31. Young D Chang, Mellar P Davis, Joshua Smith, Terrence Gutgsell (2016) Pseudobulbar Affect or Depression in Dementia?. J Pain Symptom Manage 51(5): 954-958.

32. https://www.psychcongress.com/saundras-corner/scales-screeners/ pseudobulbar-affect-pba/center-neurologic-study-liability-scale-cns, $05 / 05 / 2021$

33.https://mstrust.org.uk/a-z/pseudobulbar-affect-pathologicallaughing-and-crying

34. Roy E Strowd, Michael S Cartwright, Michael S Okun, Ihtsham Haq, Mustafa S Siddiqui (2010) Pseudobulbar affect: prevalence and quality of life impact in movement disorders. J Neurol, 257(8): 1382-1387.

35. Suzuki K, Miyamoto M, Miyamoto T, Hirata K (2015) Parkinson's disease and sleep/wake disturbances, Curr Neurol Neurosci Rep15(3): 8.

36. https://www.mayoclinic.org/diseases-conditions/pseudobulbaraffect/symptoms-causes/syc-20353737,05/08/2021

37. https://www.ajmc.com/view/pharmacotherapeutic-management-ofpseudobulbar-affect, 05/08/2021

38.https:// www.mayoclinic.org/diseases-conditions/ p se u d obulbar-affect/diagnos is - treat ment/d r c 20353741\#: :text=Antidepressants $\% 20$ for $\% 20$ the $\% 20$ treatment $\% 20$ of,designed $\% 20$ to $\% 20$ specifically $\% 20$ treat $\% 20$ PBA., 05/05/2021

39. Ahmed A, Simmons Z (2013) Pseudobulbar affect: prevalence and management. Ther Clin Risk Manag 9:483-489.

40. Miller A, Pratt H, Schiffer R. Pseudobulbar (2011) affect: the spectrum of clinical presentations, etiologies, and treatments Expert Rev Neurother 11:1077-1088.

41. Villeneuve Y, Cruz Santiago D, Masson H, Clerc D (2020) Treatment of pseudobulbar affect in a mixed neurodegenerative disorder with compounded quinidine capsules and dextromethorphan cough syrup. SAGE Open Med Case Rep 8:2050313X20921076.

42. https://www.mayoclinic.org/diseases-conditions/rem-sleep-behaviordisorder/symptoms-causes/syc-20352920, 05/14/2021.

43. https://www.mayoclinic.org/diseases-conditions/restless-legssyndrome/symptoms-causes/syc-20377168, 05/14/2021

44. Tammie Lee Demler (2017) Introduction to Pseudobulbar Affect: Setting the Stage for Recognition and Familiarity with This Challenging Disorder Am J Manag Care (18): 339-344.

45. Healthwise Staff Medical Review:E. Gregory Thompson MD - Internal Medicine \& Colin Chalk MD, CM, FRCPC - Neurology August 4, 2020

46. Jorge Garza-Ulloa (2018) Chapter 7 - Case studies of applied Biomechatronics solutions based on mathematical models,Applied Biomechatronics using Mathematical Models, Academic Press 525-626.

47. Jorge Garza Ulloa (2018) Chapter 2 - Introduction to human neuromusculoskeletal systems, Editor(s): Jorge Garza-Ulloa, Applied Biomechatronics using Mathematical Models, Academic Press 53-118.

48. Pioro EP (2011) Current concepts in the pharmacotherapy of pseudobulbar affect. Drugs 71(9):1193-1207. 